\title{
A New High-Performance Monopulse Feed with a Simple Comparator Network
}

\author{
Nyambayar Jargalsaikhan $\mathbb{D}^{1},{ }^{1}$ Jae-Hoon Bang $\mathbb{D}^{2},{ }^{2}$ Seong-Gon Choi, \\ Bierng-Chearl Ahn $\left({ }^{\oplus},{ }^{1}\right.$ and Andrii Gorshkov ${ }^{1}{ }^{1}$ \\ ${ }^{1}$ Department of Radio and Communications Engineering, Chungbuk National University, Cheongju City 26844, Republic of Korea \\ ${ }^{2}$ Duta Technology Inc., Daejeon 34077, Republic of Korea
}

Correspondence should be addressed to Jae-Hoon Bang; jhbang@duta-rnd.com and Andrii Gorshkov; andrii.gorshkov@gmail.com Received 19 October 2018; Revised 21 March 2019; Accepted 18 May 2019; Published 27 June 2019

Academic Editor: Miguel Ferrando Bataller

Copyright (C) 2019 Nyambayar Jargalsaikhan et al. This is an open access article distributed under the Creative Commons Attribution License, which permits unrestricted use, distribution, and reproduction in any medium, provided the original work is properly cited.

This paper presents a new high-performance five-element monopulse feed with a simple comparator network for Cassegrain reflector applications. The radiating element is a tapered dielectric rod fed by a circular waveguide which is in turn excited by a printed dipole. The center element is used for the sum pattern, while top and bottom elements are used for the elevation difference pattern, and left and right elements for the azimuth difference pattern. Out-of-phase excitation for the difference pattern is obtained by antisymmetrically placing two dipoles and combining their outputs with a power combiner. The optimally designed feed has been fabricated and measured. Measurement shows that the feed has the maximum sum pattern gain of $16.8 \mathrm{dBi}$, the difference pattern null depth of $-21 \mathrm{dBi}$, and the reflection coefficient of less than $-10 \mathrm{~dB}$ over 13.9-16.7 GHz.

\section{Introduction}

Monopulse antennas of single- or dual-reflector type have widely been employed for telemetry of aerial vehicles [1-3]. The feed is a key element in any monopulse reflector antenna and various designs have been proposed, which can broadly be classified into multiple-element, multiple-aperture, and multimode single aperture configurations.

In a monopulse feed, a comparator network is required to form simultaneously a sum and two difference patterns. The comparator network has usually been realized by using rectangular waveguides or printed transmission lines. Folded hybrid tees or magic tee structures are often applied to the multimode-type feed $[4,5]$. In the planar-type monopulse feed, the plate-laminated waveguide comparator can be used [6]. The above comparator structures are very complicated requiring extensive design work and high fabrication cost. Monopulse feeds employing four or five dielectric rods have been investigated in $[7,8]$. Compared to horn-type feeds, they have such advantages as structural simplicity and ease of simultaneous optimization of the sum and difference patterns.
In this letter, we present a new high-performance fivedielectric-rod monopulse feed with a simple comparator network, which is suitable for dual-reflector antenna applications. Employed in our design is a simple comparator consisting of printed dipoles, coaxial cables, and power combiners. The design and realization of the proposed feed are described in the following.

\section{Structure of the Proposed Feed}

Figure 1(a) shows the overall structure of the proposed feed employing five dielectric rods fed by a circular waveguide, which is in turn excited by a printed dipole. The dielectric rods are modelled by the polycarbonate material having a dielectric constant of 2.70 and a loss tangent of 0.0007 at 15 $\mathrm{GHz}$. For a minimum blockage of a reflector antenna, it is desirable to feed the circular waveguide with an end launcher. To this end, a coaxial probe-fed transverse wire or an axial ridge can be employed. In this paper, we employed a printed dipole for its ease of design, easy polarization rotation, possibility of dual polarizations, and precise fabrication by chemical etching. The dipole's signal path length is about 


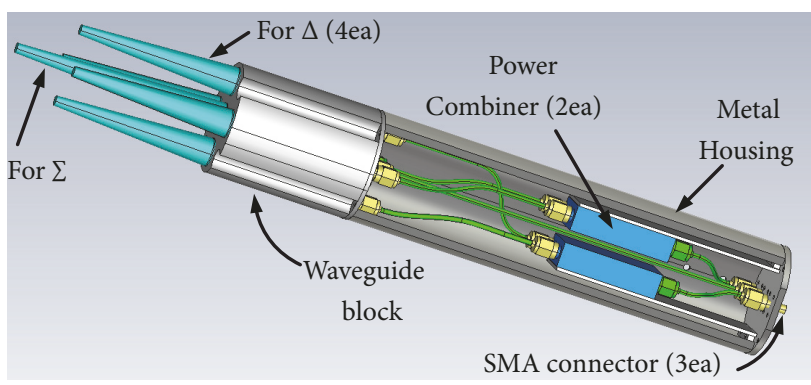

(a)

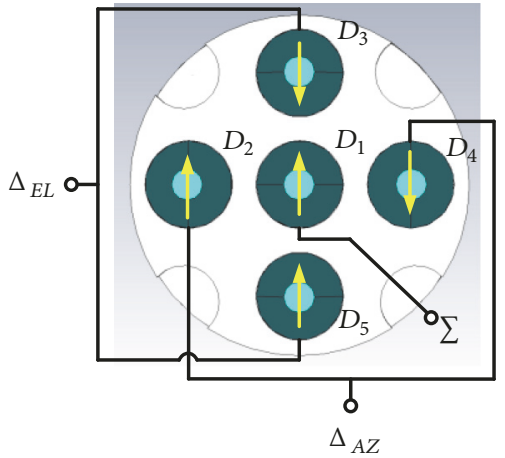

(b)

FIgURE 1: Construction of the proposed feed. (a) Overall structure. (b) Electric field direction and comparator topology.

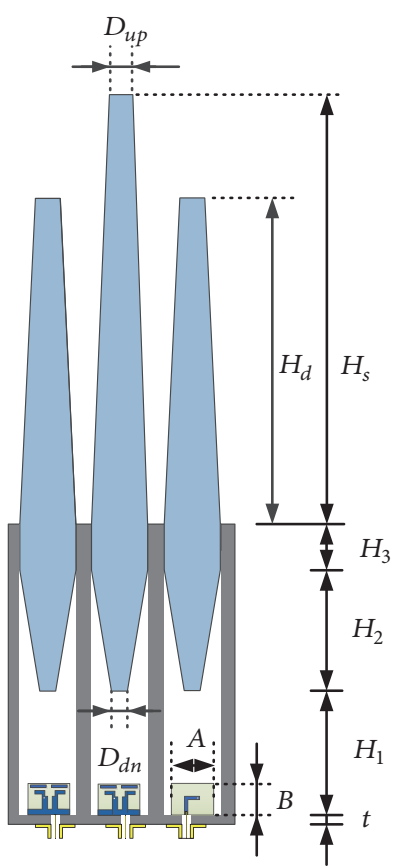

(a)

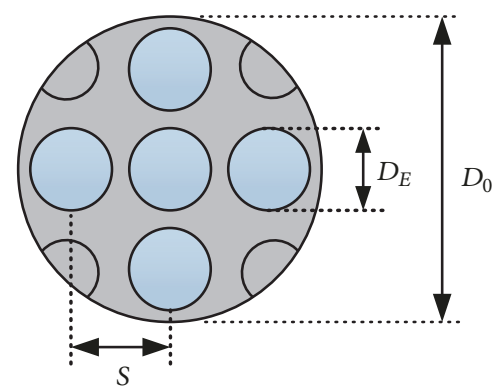

(b)

FIgURE 2: Dimensional parameters of the proposed feed. (a) Cross-sectional view. (b) Top view.

$7 \mathrm{~mm}$, for which the loss will be $0.083 \mathrm{~dB}$ for a $50 \mathrm{ohm}$ microstrip line at $15 \mathrm{GHz}$. The power-handling capability of the proposed feed is limited by the printed transmission lines and the coaxial cables.

Figure 1(b) shows the electric field directions in each radiator and the topology of the comparator network. The center rod $S$ provides a sum pattern, while the horizontal $\left(D_{2}\right.$ and $\left.D_{4}\right)$ and vertical $\left(D_{3}\right.$ and $\left.D_{5}\right)$ pairs generate the azimuth and elevation difference patterns, respectively. The $180^{\circ}$ outof-phase excitation for the difference pattern is achieved by antisymmetrically placing two corresponding dipoles as shown in Figure 1(b). The printed dipole is fed by a panelmount SMA probe connector. For the sum channel, the dipole output is routed to an SMA connector at the rear of the feed assembly. For the difference patterns, two dipoles are connected to the input of a coaxial power combiner using a pair of phase-matched coaxial cables and the combiner output is routed to the rear of the feed assembly. Coaxial cables and power combiners are placed inside a cylindrical metal housing.

Figure 2 shows dimensional parameters of the designed feed. The proposed feed is designed in the following steps. The designs of a proposed feed are carried out with the Microwave Studio $^{\mathrm{TM}}$ by CST. First, the circular waveguide diameter is chosen such that the cutoff frequency of the $\mathrm{TE}_{11}$ mode is 0.94 times the lowest operating frequency. Next, the lengths of the dielectric rods are determined for design goals of 10 $\mathrm{dB}$ taper and $8 \mathrm{~dB}$ taper in the sum and difference patterns, respectively, at the edges of illumination angle $\left( \pm 30^{\circ}\right)$. For the difference pattern, the element spacing is chosen to be a 


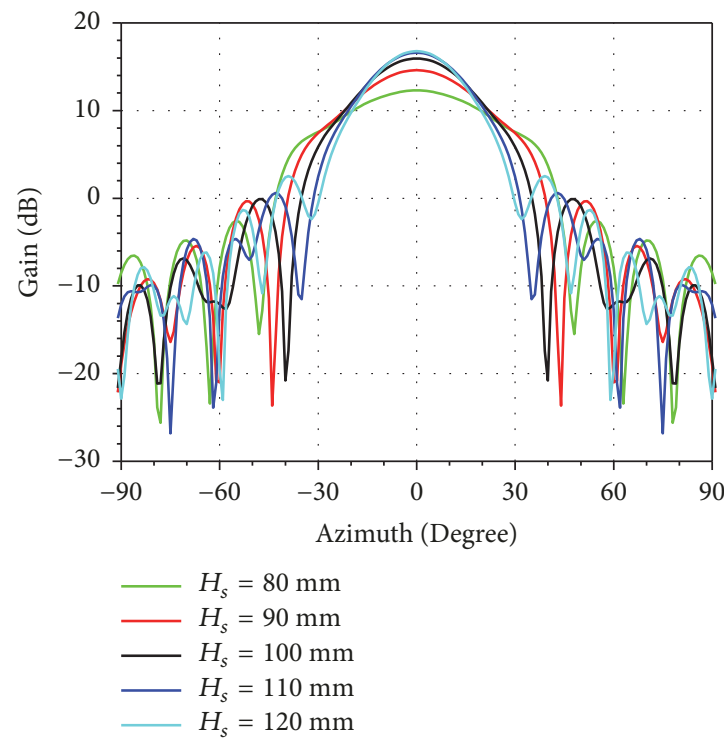

Figure 3: Sum pattern versus the center dielectric rod length.

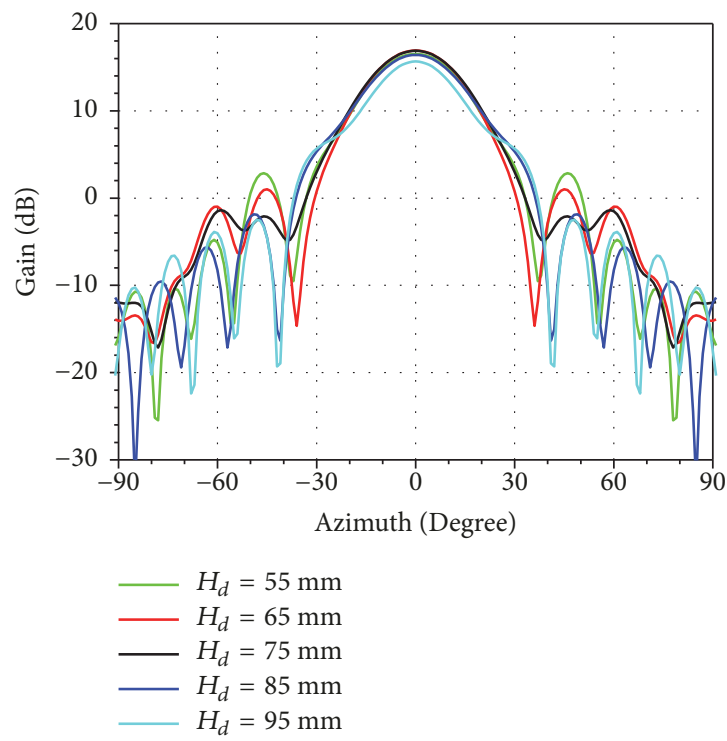

FIGURE 4: Sum pattern versus the side dielectric rod length.

minimum practical value dictated by the circular waveguide diameter.

The dielectric rod is linearly tapered over its length for impedance matching and improvements in gain and sidelobe characteristics [9]. The portion of the dielectric rod inside the waveguide is also linearly tapered for good impedance matching performances.

An optimum design of the proposed feed is obtained by adjusting the dielectric rod length. The length of dielectric rods for the difference pattern is less than that of the dielectric rod for the sum pattern to avoid its performance degradations. Given below are the dielectric rod designs at $15 \mathrm{G} \mathrm{Hz}$. The sum pattern edge taper can be adjusted by changing the center dielectric rod length $H_{s}$ as shown in
Figure 3. The length of the center dielectric rod has little effect on the difference pattern. A value of $110 \mathrm{~mm}$ is chosen for $H_{s}$.

Figure 4 shows the sum patterns versus the side dielectric rod length $H_{d}$. The sidelobes are increased when the length $H_{d}$ is less than $65 \mathrm{~mm}$. The length $H_{d}$ has little effects on the elevation and azimuth difference patterns. Figure 5 shows the effect of the dielectric rod center-to-center distance $S$ on the sum and difference patterns. We have chosen $17 \mathrm{~mm}$ for the dielectric rod distance to satisfy the aforementioned edge taper requirements.

The portion of the dielectric rod inside the circular waveguide is tapered to reduce reflection. The taper length of $30 \mathrm{~mm}$ is chosen in the design. The dielectric rod array for monopulse pattern is optimally designed based on the 


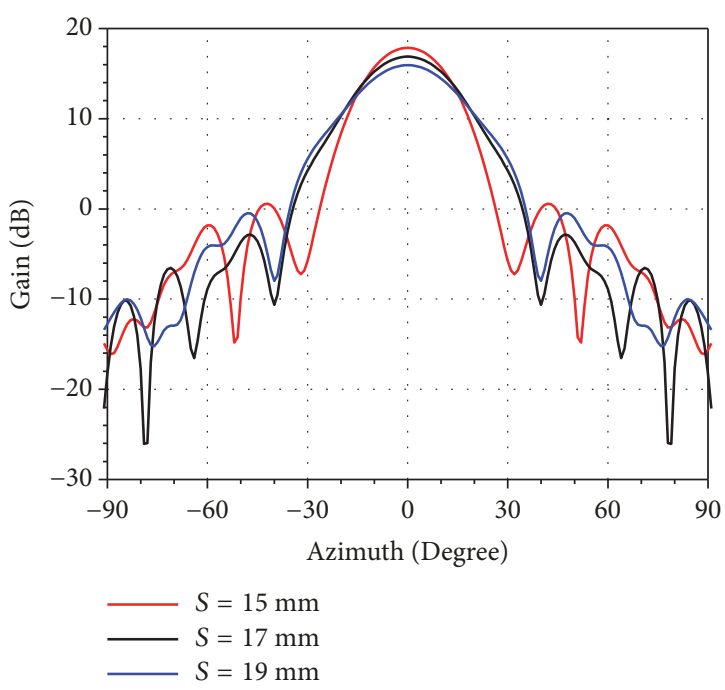

(a)

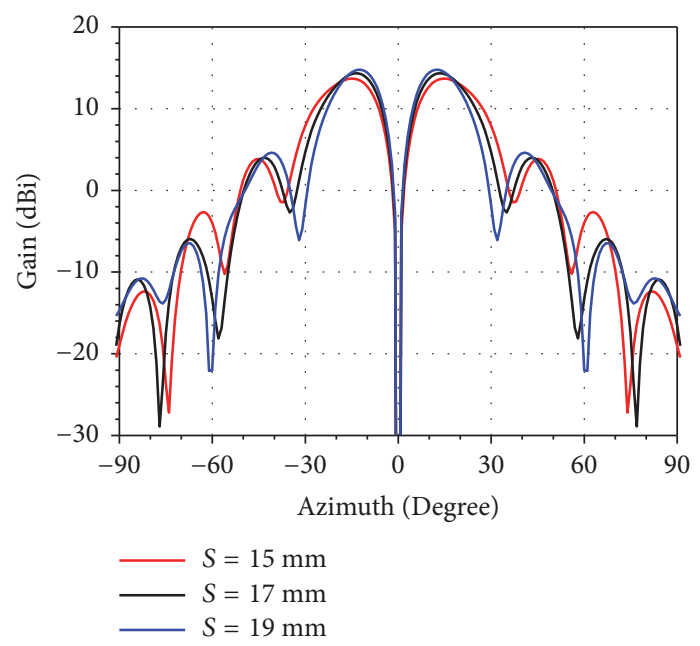

(b)

FIGURE 5: Gain patterns versus the dielectric rod spacing. (a) Sum pattern. (b) Elevation difference pattern.

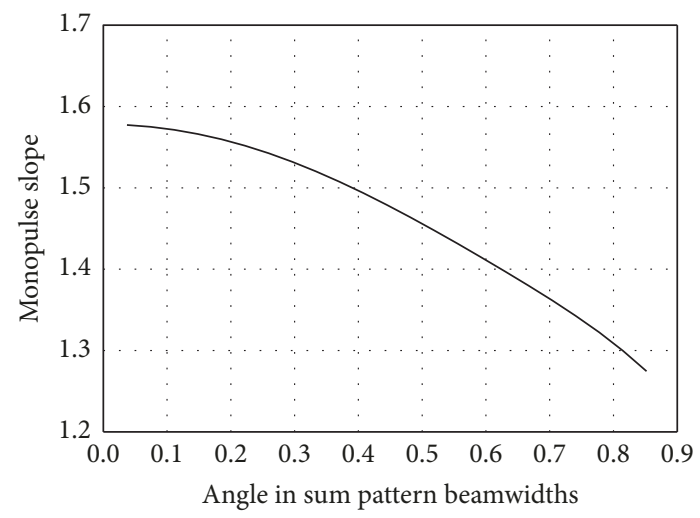

FIGURE 6: Monopulse slope of the desgined feed.

parameter study described. The dimensions in millimeter of the optimally design dielectric rods operating at $15 \mathrm{GHz}: D_{0}$ = 54.0, $D_{E}=13.4, S=17.0, H_{s}=110.0, H_{d}=75.0, H_{1}=67.0, H_{2}$ $=30.0, H_{3}=10.0, D_{u p}=4.7, D_{d n}=3.6$, and $t=2.0$.

Sum and difference patterns of the optimally designed feed will be presented in Section 3 along with the measurement. The feed's difference pattern slope will affect the Cassegrain reflector's tacking accuracy. We have calculated the normalized monopulse slope $k_{m}$ in volt per volt per beamwidth, plotted in Figure 6. Consider the following:

$$
k_{m}=\frac{d G_{\Delta}}{d \theta} \frac{\theta_{3 \mathrm{~dB}, \mathrm{sum}}}{G_{\text {sum }}}
$$

where $\theta$ is the pattern angle, $G_{\Delta}$ is the difference pattern gain, $G_{\text {sum }}$ is the sum pattern gain, and $\theta_{3 \mathrm{~dB} \text {,sum }}$ is the sum pattern half-power beamwidth. The monopulse slope of the feed ranges from 1.58 to 1.45 when the angle varies from zero to half the sum pattern $3 \mathrm{~dB}$ beamwidth.
With the design of radiating elements accomplished, the next step is to design a printed dipole for the excitation of the circular waveguide. Figure 2(a) shows circular waveguides excited by a printed dipole. The backshort in the waveguide serves as the ground plane for the printed dipole and a coaxial probe feeding the dipole. Figure 7 shows the circuit pattern and dimensional parameters of the printed dipole. The dipole is fed by a microstrip line with an integrated balun [10]. The input microstrip line of the printed dipole is fed by a coaxial probe on the circular waveguide backshort which serves as a ground plane for the probe. The coaxial cable is behind the backshort. With this arrangement, one does not need a balun on the coaxial cable.

The printed dipole is realized on the Isola I-Tera MT RF substrate of a thickness $0.508 \mathrm{~mm}$, a dielectric constant 3.45, and a loss tangent 0.0031 . The microstrip line is in turn fed by a coaxial probe. Two parasitic strips on top of the dipole arms are employed to increase the VSWR bandwidth from about $10 \%$ to $18 \%$.

A printed dipole exciting a $13.4 \mathrm{~mm}$ circular waveguide is designed starting from initial theoretical design and final design is achieved by optimization procedure provided in the simulation software Microwave Studio ${ }^{\mathrm{TM}}$. Dimensions (in $\mathrm{mm}$ ) of the designed printed dipole are as follows: $A=8.00$, $B=6.59, a_{1}=0.85, a_{2}=2.91, a_{3}=0.87, a_{4}=0.46, a_{5}=0.7, a_{6}$ $=2.64, a_{7}=1.73, a_{8}=0.29, a_{9}=2.86, a_{10}=0.91, a_{11}=2.45, b_{1}$ $=1.30, b_{2}=2.40, b_{3}=1.08, b_{4}=0.59, b_{5}=0.56, b_{6}=0.66, b_{7}$ $=2.31, c_{1}=3.78, c_{2}=2.96, w=0.55, v_{x}=0.61$, and $v_{y}=3.51$.

Next, five dipoles exciting the circular waveguide are combined in a monopulse comparator network to obtain the sum, azimuth difference, and elevation difference patterns. The complexity of the monopulse comparator network lies in the fact that combiner networks for sum, azimuth difference, and elevation difference channels cross each other. To simplify the problem of transmission line routing, we have employed coaxial transmission lines and coaxial power 


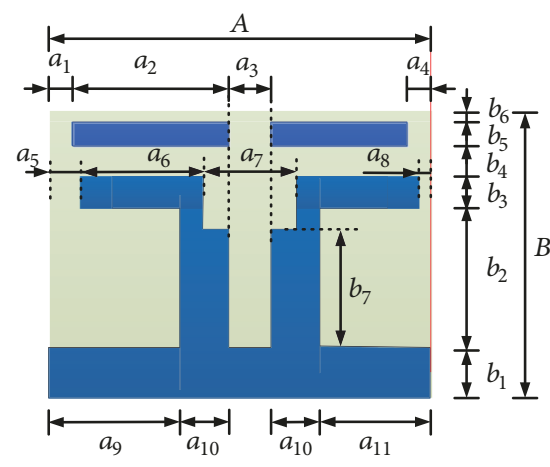

(a)

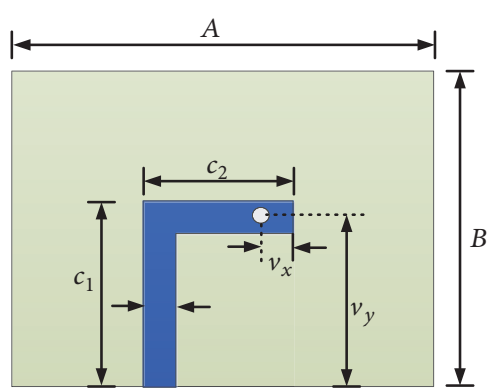

(b)

Figure 7: Dimensional parameters of the printed dipole on the (a) front side and (b) back side.

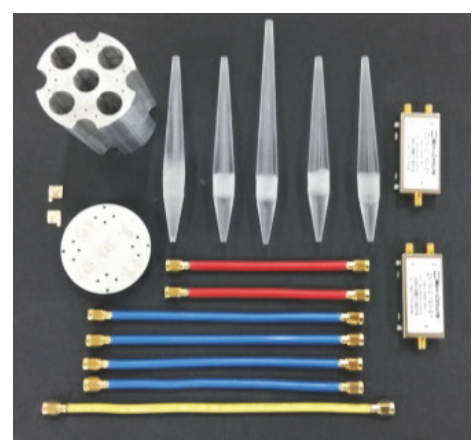

(a)

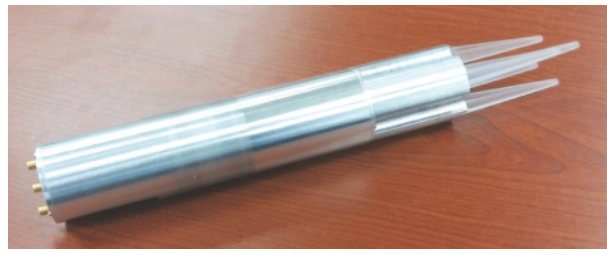

(b)

Figure 8: (a) Feed components and (b) the fabricated feed assembly.

combiner as shown in Figure 1 . The often-used $180^{\circ}$ outof-phase power combiner is not required in our design. The task is carried out by placing the two-dipole pair in an opposite direction. A low-cost commercial off-the-shelf power combiner is employed in combining two dielectric rod radiators to form a difference pattern. Lengths of coaxial cables can easily be trimmed so that the sum, azimuth, and elevation difference channel all have the same physical as well as electrical length when the channels need to be combined to form single-channel monopulse architecture.

\section{Fabrication and Measurement}

The designed feed has been fabricated and tested. The plastic dielectric rod and the waveguide aluminum block have been fabricated by numerically controlled machining with an accuracy of $\pm 0.02 \mathrm{~mm}$. The printed dipole has been fabricated using standard photolithographic printed circuit fabrication processes.

Figure 8 shows the fabricated feed. Specified lengths of the RG-402 cable assembly operating up to $20 \mathrm{GHz}$ and commercial off-the-shelf coaxial power combiner/divider by Mini-Circuits (ZX10- 2-183-S+) have been employed to implement the monopulse comparator network. A typical RG-402 cable has loss of about $1.5 \mathrm{~dB} / \mathrm{m}$ at $15 \mathrm{GHz}$. A lowloss equivalent to RG-402 has $0.9 \mathrm{~dB} / \mathrm{m}$ at $15 \mathrm{GHz}$. The length of the cables employed in our design is about $15 \mathrm{~cm}$ at the maximum resulting in $0.23 \mathrm{~dB}$ loss. With a low-loss cable, loss will be $0.14 \mathrm{~dB}$. The sum, azimuth, and elevation difference channel outputs come out of SMA connectors at the back face of the feed.

Figure 9 shows measured gain patterns at $15 \mathrm{GHz}$ of the sum and azimuth difference channels measured in an anechoic chamber far-field range. The elevation difference pattern (not shown) is similar to that of the azimuth difference channel. The measured sum pattern has maximum gain of $16.8 \mathrm{dBi}$, and taper levels of about $-10 \mathrm{~dB}$ at $\pm 25^{\circ}$ off the boresight, for which a Cassegrain reflector optics can be designed. If necessary, we can reduce the $10 \mathrm{~dB}$ taper angle to $\pm 20^{\circ}$ by increasing the length of the dielectric rods. The measured difference pattern has maximum gain of $14.2 \mathrm{dBi}$, null gain of $-21.0 \mathrm{dBi}$, and taper levels of about $4.5 \mathrm{~dB}$ at $\pm 25^{\circ}$ off the boresight. Gain values beyond $\pm 90^{\circ}$ monotonically decrease. The front-to-back ratio in the sum pattern is about $35 \mathrm{~dB}$. Measurements agree well with calculations.

The reflection coefficient of the fabricated feed has been measured using the HP8720C network analyzer and is shown in Figure 10. It is less than $-10 \mathrm{~dB}$ at $13.5-16.9 \mathrm{GHz}$ for the sum channel and less than $-10 \mathrm{~dB}$ at $13.9-16.7 \mathrm{GHz}$ for the difference channels. The simulated reflection coefficient of the feed is not included in Figure 10 since it involves coaxto-microstrip transitions, coaxial connectors, and power combiners. 


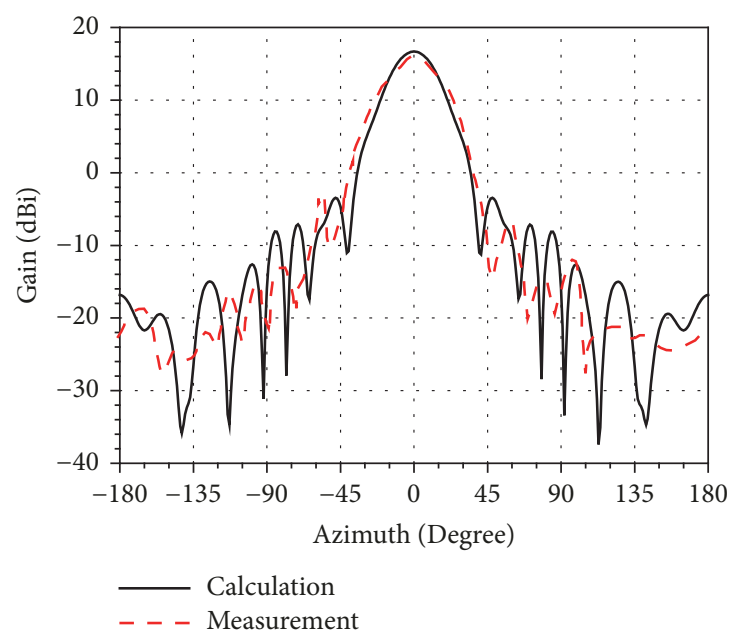

(a)

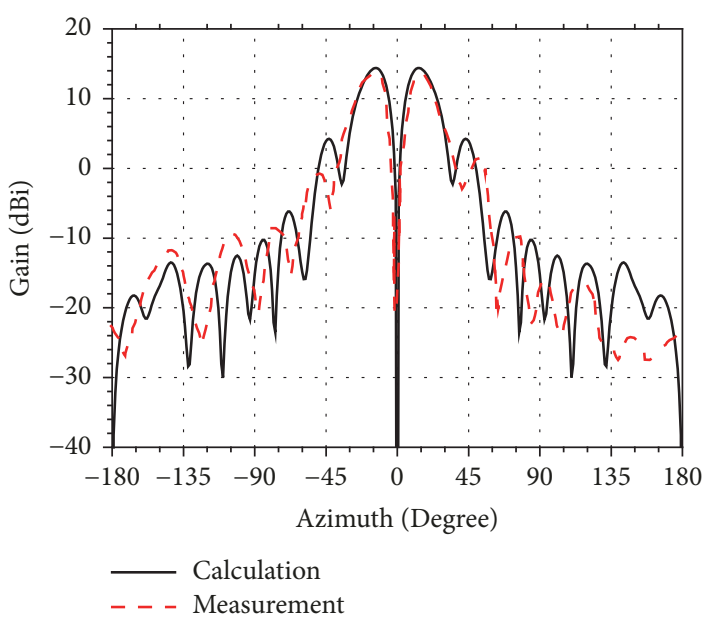

(b)

FIGURE 9: Measured gain patterns of the fabricatd feed. (a) Sum pattern. (b) Azimuth difference pattern.

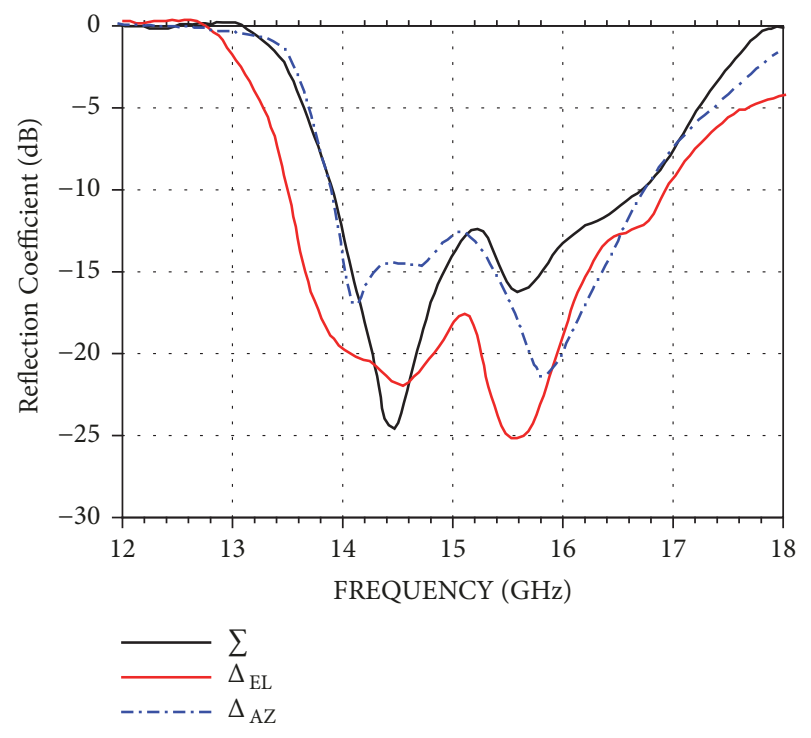

FIGURE 10: Measured reflection coefficient of the fabricated feed.

\section{Conclusion}

In this paper, we presented a new high-performance monopulse feed employing five dielectric rods with a simple comparator network consisting of two power combiners and coaxial cables. Simplification of the monopulse comparator has been made possible by antisymmetric excitation of two dielectric rod elements forming a difference pattern. The measurement of the fabricated feed has shown that the proposed feed has such desirable characteristics as simultaneous optimum illumination of the Cassegrain subreflector over $\pm 25^{\circ}$, null depth of $37.4 \mathrm{~dB}$ relative to the maximum sum pattern gain and VSWR bandwidth of $18 \%$. Although a design is presented for a Cassegrain reflector application, the proposed feed can also be used as a stand-alone monopulse antenna with sum channel gain up to $20 \mathrm{~dB}$ by properly designing the dielectric rod radiator.

\section{Data Availability}

The data used to support the findings of this study are included within the article.

\section{Conflicts of Interest}

The authors declare that there are no conflicts of interest regarding the publication of this paper.

\section{Acknowledgments}

This work was supported by "Human Resources Program in Energy Technology" of the Korea Institute of Energy Technology Evaluation and Planning (KETEP) and was granted financial resource from the Ministry of Trade, Industry \& Energy, Republic of Korea (no. 20164030201330).

\section{References}

[1] S. M. Sherman, Monopulse Principles and Techniques, Artech House, Dedham, Mass, USA, 1984.

[2] T. Bhuiya, R. Harsh, and K. Tuckley, "Microstrip monopulse feed for parabolic dish tracking antenna used in a radio theodolite system," IETE Technical Review, vol. 27, no. 2, pp. 120-123, 2010.

[3] A. Tribak, A. Mediavilla, K. Cepero, and J. L. Cano, "Highly efficient monopulse tracking feed subsystem for unmanned aerial vehicle," in Proceedings of the Proc. 41st Euro. Microw. Conf, pp. 1027-1030, Manchester, UK, 2011.

[4] K. M. Lee and R.-S. Chu, "Design and analysis of a multimode feed horn for a monopulse feed," IEEE Transactions on Antennas and Propagation, vol. 36, no. 2, pp. 171-181, 1988. 
[5] H. Kumar, G. Kumar, Y. Verma, and P. K. Mishra, "Compact waveguide monopulse comparator at Ka-band for monopulse tracking," in Proceedings of the 2016 IEEE International Symposium on Antennas and Propagation \& USNC/URSI National Radio Science Meeting, pp. 1357-1358, Fajardo, Puerto Rico, June 2016.

[6] X. Xu, J. Hirokawa, and M. Ando, "Double-layered platelaminated waveguide monopulse comparator in E-band," in Proceedings of the 2016 IEEE International Symposium on Antennas and Propagation \& USNC/URSI National Radio Science Meeting, pp. 1703-1704, Fajardo, Puerto Rico, June 2016.

[7] S. Qian, X. Li, and B. Wang, "Ka band Cassegrain monopulse antenna fed by tapered rod antennas," in Proceedings of the 2008 8th International Symposium on Antennas, Propagation and EM Theory, pp. 39-41, Kunming, China, November 2008.

[8] C. Kumar, V. S. Kumar, and V. V. Srinivasan, "Design aspects of a compact dual band feed using dielectric rod antennas with multiple element monopulse tracking," IEEE Transactions on Antennas and Propagation, vol. 61, no. 10, pp. 4926-4932, 2013.

[9] T. Ando, I. Ohba, S. Numata, J. Yamauchi, and H. Nakano, "Linearly and curvilinearly tapered cylindrical-dielectric-rod antennas," IEEE Transactions on Antennas and Propagation, vol. 53, no. 9, pp. 2827-2833, 2005.

[10] B. Edward and D. Rees, "Broadband printed dipole with integrated balun," Microwave Journal, vol. 30, no. 5, pp.339-344, 1987. 


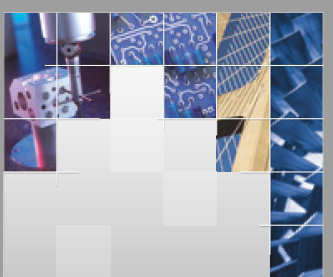

\section{Enfincering}
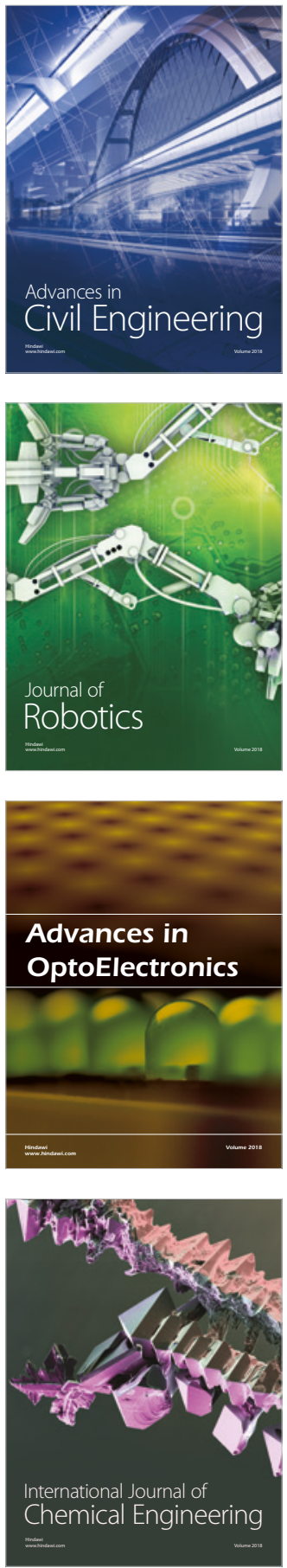

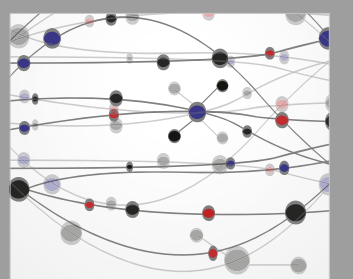

\section{Rotating \\ Machinery}

The Scientific World Journal

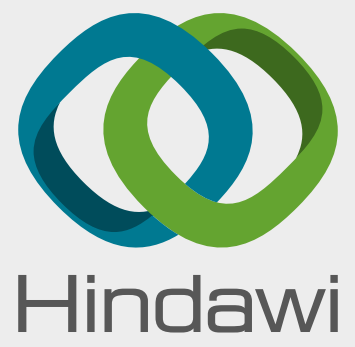

Submit your manuscripts at

www.hindawi.com
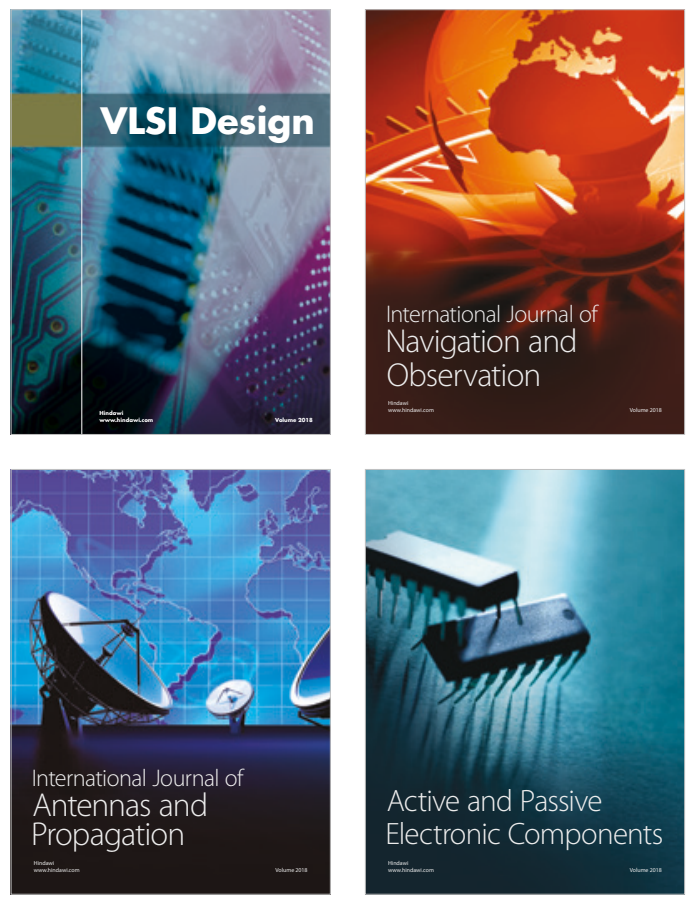
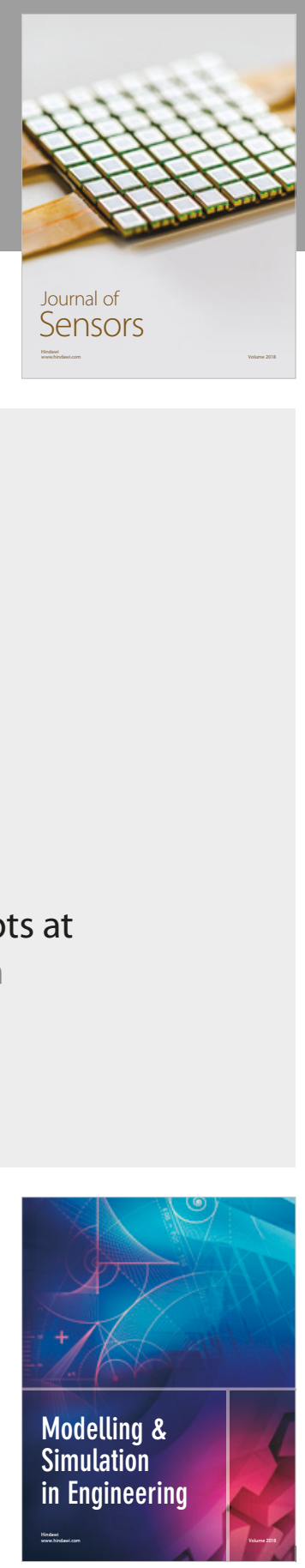

\section{Advances \\ Multimedia}
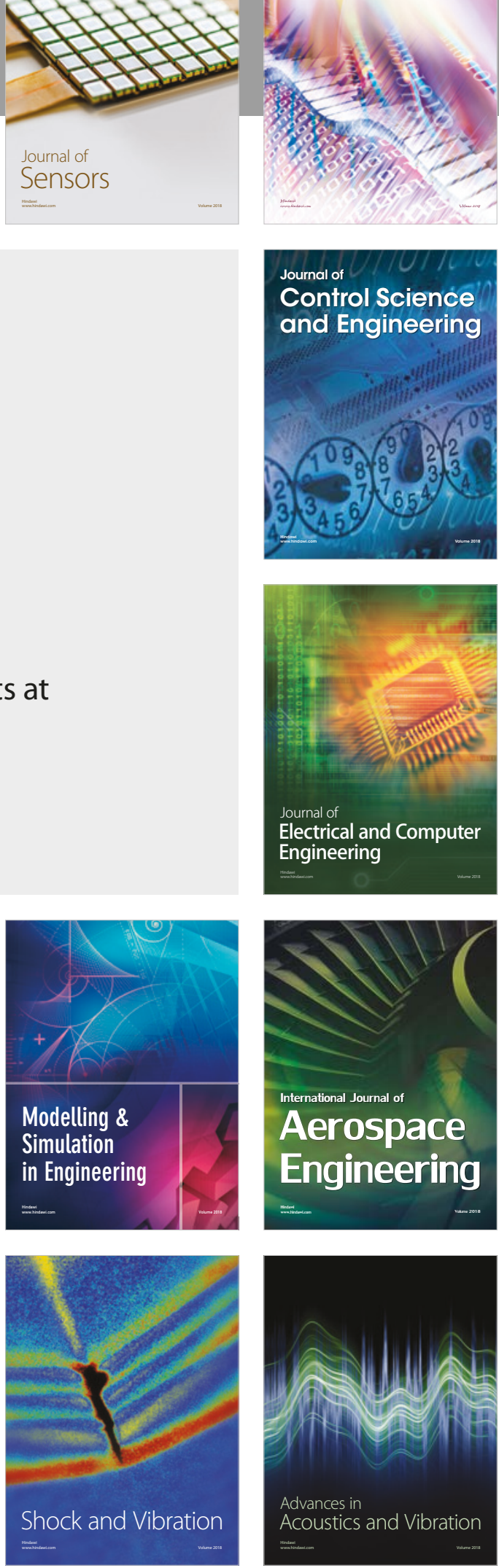